\title{
Memoria e inteligencia: interdependencia en función de los procesos y contenidos de las tareas
}

\author{
Memory and intelligence: Interdependence \\ according to processes and content of tasks
}

Recibido: mayo 25 de 2010 | Revisado: noviembre 20 de 2010 | Aceptado: diciembre 14 de 2010

\author{
ARISTIDES I. FERREIRA** \\ ISCTE - Instituto Universitário de Lisboa, Portugal \\ LEANDRO S. AlMEIDA **** \\ Universidade do Minho, Portugal \\ GERARDO PRIETO **** \\ Universidad de Salamanca, España \\ M. ADELINA GUISANDE ${ }^{* * * * *}$ \\ Universidad de Santiago de Compostela, España
}

SICI: 1697-9267(201206)11:2<455:MEIIFP>2.0.TX;2-Z

Para citar este artículo. Ferreira, A. I., Almeida, L. S., Prieto, G. \& Guisande, M. A. (2012). Memoria e inteligencia: interdependencia en función de los procesos y contenidos de las tareas. Universitas Psychologica, 11(2), 455-467.

* Artículo de investigación. Este trabajo fue parcialmente financiado por la Fundação para a Ciência e a Tecnologia (Pest-OE/EGE/UI0315/2011).

** ISCTE- Instituto Universitário de Lisboa, Business Research Unit, Room D415 / Box: 320, Av. Das Forças Armadas 1649-026 Lisboa, Portugal. E-mail: aristides.ferreira@iscte.pt

**** Instituto de Educação, Universidade do Minho. Campus de Gualtar, 4715, Braga, Portugal. E-mail: leandro@ie.uminho.pt. ResearcherID: Almeida, L. B-3040-2011

****** Facultad de Psicología, Universidad de Salamanca, Avenida de la Merced, 109-131, 37005 Salamanca, España. E-mail: gprieto@usal.es. ResearcherID: Prieto, G. F-3397-2012.

******* Facultad de Psicología, C/ Xosé María Suárez Núñez, s/n. Campus Vida 15782, Santiago de Compostela, España. E-mail: mariaadelina. guisande@usc.es. ResearcherID: Guisande, M. A. F-3456-2012.

\section{RESUMEN}

Este trabajo procura analizar las características de los factores específicos de la memoria (procesos o contenidos) asociados a Gsm del modelo CHC. Para esto, a una muestra de estudiantes universitarios se le administraron tareas de memoria con diferentes contenidos, varios tests de aptitudes y una prueba de inteligencia general. Se evaluaron dos modelos que relacionan la aptitud general Gsm, bien con la memoria de trabajo y la amplitud de memoria, bien con aptitudes verbales-numéricas y viso-espaciales. Los resultados muestran, para ambos modelos, buenos índices de ajuste, sin embargo, se encontraron datos de regresión estandarizados con una regresión positiva superior entre Gsm y memoria de trabajo y entre Gsm y contenido viso-espacial. También se debe destacar que en ambos modelos se observaron valores de regresión estandarizados de 0.54 y 0.73 entre Gsm y el factor $g$. Teniendo en cuenta los resultados obtenidos, se discuten algunas implicaciones del estudio para comprender la relación entre inteligencia y memoria.

Palabras clave autores

Memoria de trabajo, amplitud de memoria, inteligencia general, procesos, contenidos.

Palabras clave descriptores

Psicometría, modelo de Rasch, aptitud espacial.

\section{A B S T R A C T}

This study investigates the characteristics of the specific memory factors (processes and content) that are associated to the Gsm from the CHC model. Memory tasks with different content, including various aptitude tests and a general intelligence test, were administered to a sample of university students. Two models that relate the general Gsm aptitude, with working memory and memory span, and with verbal-numerical and visual-spatial aptitudes were tested. Results indicate good fit indices for both models tested, as well as evidence for a positive regression between Gsm and working memory and between Gsm and visuospatial content. In both models we observed standardized regression of 0.54 and of 0.73 between Gsm and factor g. Implications for the understanding of the relation between memory and intelligence are discussed.

Key words authors

Working memory, memory span, general intelligence, processes, content.

Key words plus

Psychometry, Rasch model, spatial aptitude. 


\section{Introducción}

A lo largo de la primera mitad del siglo XX, en el estudio de la inteligencia humana han surgido diferentes polémicas, entre ellas las relacionadas con su estructura. La discusión se ha centrado en el número de factores que componen la inteligencia y sobre cómo se organizan. En este contexto, surgen los defensores de un factor general ( $g$ ) de inteligencia, en la línea teórica de Spearman $(1904,1927)$, y los defensores de una inteligencia multidimensional y constituida por aptitudes autónomas entre sí (Guilford, 1967; Thurstone, 1938).

En la actualidad, esta controversia se ha superado satisfactoriamente con la aparición de modelos que defienden una organización jerárquica de las capacidades mentales constituyentes de la inteligencia humana (Almeida, Guisande \& Ferreira, 2009). Así, hoy por hoy, el modelo teórico CHC (Cattell-Horn-Carroll) sobre las aptitudes cognitivas se asume como una referencia inevitable en la definición y evaluación de la inteligencia. Este modelo es fruto de la integración de la teoría sobre la "inteligencia fluida-inteligencia cristalizada" (GfGc) de Cattell-Horn (Horn \& Cattell, 1966; Horn \& Noll, 1997) con la teoría de los tres estratos de Carroll (Carroll, 1993). Partiendo de un conjunto de análisis factoriales, el modelo propone una estructura jerárquica, en la que se disponen las capacidades mentales, compuesta por una serie amplia de aptitudes específicas de nivel inferior o de primer orden (Stratum I), como la velocidad para acceder a la palabras, el reconocimiento de patrones, la manipulación de imágenes o la capacidad para recordar un conjunto específico de palabras, letras o números; un grupo de aptitudes de nivel intermedio o de segundo orden (Stratum II) que explicarían las relaciones entre los factores del primer nivel, como el conocimiento, el razonamiento viso-espacial, la velocidad de procesamiento o la capacidad para razonar; y una aptitud de nivel superior o de tercer orden, asociada al factor general o factor $g$ (Stratum III), como capacidad intelectual más general y básica a todas las funciones cognitivas.

En el marco del modelo de Cattell-Horn-Carroll, la memoria (en términos de memoria a corto plazo) surge representada en el nivel intermedio por Gsm. Este factor se define como la capacidad para mantener la información y recuperarla en cortos períodos de tiempo. Asociadas a Gsm, el modelo considera como aptitudes de nivel inferior (Stratum I) la amplitud de memoria (MS, memory span) y la memoria de trabajo (WM, working memory). La amplitud de memoria es la capacidad para codificar, almacenar y recuperar la información que se mantiene temporalmente en la memoria, mientras que la memoria de trabajo es la capacidad para almacenar temporalmente la información y ejecutar un conjunto de operaciones cognitivas de gestión de los procesos atencionales, perceptivos y mnémicos.

Diversos estudios diferencian los constructos amplitud de memoria y memoria de trabajo en términos cognitivos (Bayliss, Jarrold, Gunn \& Baddeley, 2003; Kane et al., 2004). La amplitud de memoria aparece como un constructo más específico que la memoria de trabajo (Engle, Tüholski, Laughlin \& Conway 1999; Kane et al., 2004). A pesar de esto, los resultados de diferentes análisis factoriales confirmatorios permiten afirmar que amplitud de memoria y memoria de trabajo son dos variables latentes correlacionadas entre sí (cerca de 0.68 en el estudio de Engle et al., 1999). En el modelo de Cattell-Horn-Carroll, como hemos mencionado, la aptitud Gsm aparece descrita por los procesos implicados, tanto a nivel de almacenamiento (MS, amplitud de memoria), como de ejecución cognitiva (WM, memoria de trabajo).

Por su parte, los modelos jerárquicos de inteligencia (e.g., Carroll, 1993; Cattell, 1971; Horn \& Noll, 1997; Vernon, 1961) destacan también factores intermedios en el desempeño cognitivo (Stratum II) asociados al contenido de las tareas (verbalauditiva, cuantitativa-numérica, viso-espacial). Las diferencias individuales en el procesamiento cognitivo en función del contenido de las tareas podrían estar relacionadas con el funcionamiento del cerebro humano, con el background experiencial de los sujetos, con sus opciones académicas y sus motivaciones. En esta línea, la investigación sugiere diferencias en el procesamiento de la memoria de trabajo en función del contenido verbal o no verbal 
de la información (Engle, Kane \& Tüholski, 1999; Ferreira, 2009; Ferreira, Almeida \& Prieto, 2011), evidenciando la existencia de variables latentes asociadas a tal procesamiento con contenido diferenciado (Shah \& Miyake, 1996). Estos estudios apoyan el modelo de los múltiples componentes de la memoria de trabajo (Baddeley \& Hitch, 1974), en el cual, junto con un ejecutivo central, se señalan dos dominios específicos representados por los componentes viso-espacial y fonológico. El ejecutivo central desempeña funciones asociadas a los procesos de atención y gestión de los recursos cognitivos, mientras que el componente viso-espacial se asocia al almacenamiento y procesamiento de la información viso-espacial, y el componente fonológico realiza el mismo proceso pero con contenido verbal.

En la actualidad, diversos autores sugieren que la memoria de trabajo se puede entender como un "homúnculo" de la cognición humana que lo explica todo. Esta afirmación surge respaldada por estudios que muestran una acentuada asociación entre la memoria de trabajo y la inteligencia general (Colom, Abad, Rebollo \& Shih, 2005; Colom, Flores-Mendonza \& Rebollo, 2003; Colom, Rebollo, Palácios, Juan-Espinosa \& Kyllonen, 2004; Colom, Rubio, Shih \& Santacreu, 2006; Kane et al., 2004; Unsworth \& Engle, 2005). Sin embargo, a pesar de que se observa un relativo consenso sobre la relación entre memoria de trabajo e inteligencia, existen grandes discrepancias en la magnitud de las correlaciones obtenidas entre los dos constructos. En este sentido, Ackerman, Beier y Boyle (2005) decidieron desarrollar un meta-análisis basado en un conjunto de estudios que relacionan memoria de trabajo e inteligencia, cuyos resultados mostraron que la media de las correlaciones (con las muestras ponderadas) es de 0.48. Los autores sugieren así, que contrariamente a las conclusiones de Kyllonen y Christal (1990), memoria de trabajo no es lo mismo que $g$ (Ackerman et al., 2005). Análisis posteriores (Martínez \& Colom, 2009; Oberauer, Schulze, Wilhelm \& Süß, 2005) muestran que Ackerman y sus colaboradores han subestimado la relación entre Gf y memoria de trabajo, mientras que Kyllonen y
Christal sobrevaloraron esta correlación. Por su parte, Schweizer (2007) critica los aspectos metodológicos de las conclusiones extraídas en estos estudios al no eliminar el porcentaje de varianza que informa de los errores asociados a las medidas utilizadas. Sin embargo, y aunque hablemos de correlaciones expresivas y estadísticamente significativas entre memoria de trabajo e inteligencia, es difícil reducir el tradicional sentido del factor $g$ como capacidad de entender, establecer relaciones y buscar correlatos (Spearman, 1927), a la coordinación de los procesos atencionales y perceptivo-conceptuales atribuidos a la memoria de trabajo. Hay procesos cognitivos superiores propios de $g$ o $g f$, en particular de relacionamiento de información, de abstracción o de evaluación de alternativas de respuesta, que difícilmente permiten su reducción a memoria de trabajo (Almeida, 1994; Garlick \& Sejnowski, 2006; Jensen, 1998; Sternberg, 1977).

Si Jensen (1973) siempre diferenció los procesos básicos de índole perceptiva y mnémica frente a los procesos cognitivos superiores encargados de la formación de conceptos, el razonamiento y la resolución de problemas; también Sternberg (1977) en su teoría componencial de la inteligencia (donde aproxima las tareas de razonamiento al factor $g$ de inteligencia), defiende que en la resolución de tareas de clasificación, analogías y silogismos interfieren, además del componente de codificación de la información y su almacenamiento en la memoria a corto plazo (encoding), un amplio conjunto de otros componentes de inferencia, correspondencia y aplicación de relaciones (inference, mapping, application), así como de comparación, justificación y evaluación de las alternativas de respuestas.

Asimismo, en un artículo reciente, Gläscher et al. (2010) parecen demostrar una autonomía neurológica del factor $g$ frente a la memoria de trabajo, basada en una diversidad de regiones encefálicas que surgen implicadas en la emergencia de un factor general de inteligencia, considerando indicadores del funcionamiento neurológico en sujetos con lesiones cerebrales. Los autores mostraron que una lesión en la región frontal izquierda [área de Brodmann (BA)10] afectaba solamente a 
la ejecución en tests de factor $g$ y no en los diversos subtests del WAIS. A pesar de estos resultados, este mismo estudio señaló fuertes evidencias que sugieren que la memoria de trabajo y el factor $g$ comparten funciones ejecutivas en el área del giro frontal inferior izquierdo y en el córtex parietal. En esta misma línea de investigación, Colom, Jung y Haier (2007) han demostrado la existencia de una estructura neurológica común asociada al giro frontal superior derecho y al giro frontal medio izquierdo (también en el área de Brodmann). Estos estudios asumen una mayor dependencia de la memoria de trabajo del factor $g$, que a la inversa. Parece existir un mecanismo común a todas las áreas asociadas a la recuperación de la información (Garlick \& Sejnowski, 2006). A pesar de esto, debemos salvaguardar el hecho de que la relación entre memoria de trabajo y factor $g$ puede ocurrir, tal vez, porque las tareas que evalúan los procesos en cuestión miden las mismas áreas del sistema nervioso (Prabhakaran, Smith, Desmond, Glover \& Gabrieli, 1997).

Teniendo en cuenta cierta controversia establecida sobre los índices y los significados de las correlaciones entre tareas de memoria y de inteligencia, así como asociaciones consistentes entre amplitud de memoria y memoria de trabajo en función de los contenidos viso-espaciales y verbales (Colom \& Flores-Mendoza, 2006), este estudio pretende analizar la convergencia de ambos constructos, cruzando las medidas de memoria con tres tests de inteligencia: un test de inteligencia general ([D48], Anstey, 1983) y dos subtests del PMA de Thurstone y Thurstone (1997), que evalúan aptitudes con alguna asociación con los contenidos de las pruebas de memoria utilizadas en este estudio: PMA-V, centrada en el contenido verbal, y el PMA-E, centrada en el contenido espacial. Dadas las características de las tareas de memoria utilizadas, se pretende también analizar si la aptitud intermedia de Gsm de la teoría de Cattell-Horn-Carroll (CHC) se manifiesta mejor a través de distintos procesos (amplitud de memoria y memoria de trabajo) o de diferentes contenidos (verbales y no verbales) en su relación con la inteligencia.

\section{Método}

\section{Participantes}

Este estudio fue realizado con 72 estudiantes universitarios, de los cuales 48 eran de sexo femenino. La media de edad fue de 27.7 años ( $\mathrm{Min}=18$; $\operatorname{Max}=46 ; D E=5.56)$. Por razones de accesibilidad, gran parte de estos alumnos estudiaban en una universidad privada (65.3 \%) de la región de Lisboa y asistían a las titulaciones de Arquitectura y Diseño (61.3 \%). Los demás estudiantes estaban matriculados en las titulaciones de Filología, Ciencias de la Educación y Psicología. El hecho de que los estudiantes de Arquitectura y Diseño cursasen la modalidad de enseñanza nocturna, explica la media elevada de las edades en la presente muestra de universitarios.

\section{Instrumentos}

En este estudio, se utilizó una batería informatizada de pruebas de memoria (Ferreira, 2009) que permite una evaluación de los constructos memoria de trabajo y amplitud de memoria, tanto de contenidos verbales como no verbales. Estudios previos (Ferreira, 2009) muestran las buenas propiedades psicométricas de las diferentes pruebas, oscilando los índices de consistencia interna entre 0.72 y 0.89 , y con un ajuste adecuado de los ítems en cada prueba (modelos de Rasch para créditos parciales e ítems dicotómicos, este último, en el caso de la tarea de Flechas y Números).

Amplitud de memoria - Dígitos: tarea próxima de la Secuencia de Números de las escalas de Wechsler (2004) en la que se presentan durante un breve período de tiempo $(1000 \mathrm{~ms})$ secuencias aleatorias de números. Además de los dos ejercicios de entrenamiento, la tarea integra 18 ítems con series de tres secuencias, del mismo tamaño, entre cuatro y diez dígitos.

Amplitud de memoria - Espacial: Basándose en los cubos de Corsi (Milner, 1971) y en una tarea desarrollada por Ichikawa (1983), se muestra durante un breve período de tiempo (1000 ms) una secuencia aleatoria de cuadrados en una matriz 6 


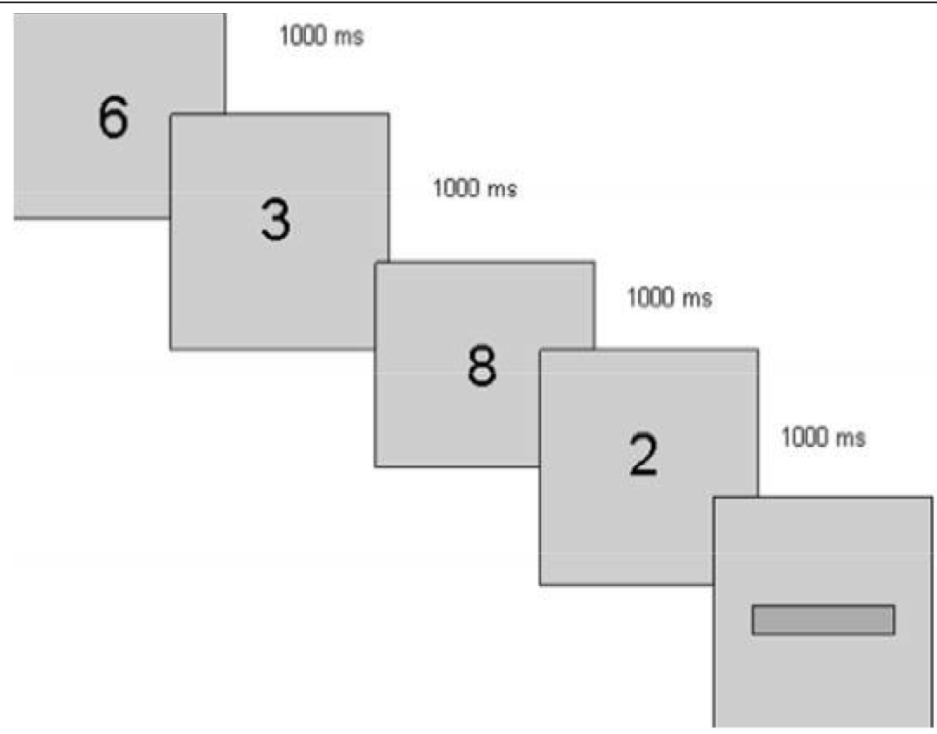

Figura 1. Ejemplo de un ítem de la tarea de Dígitos. Fuente: Ferreira (2009).

x 6 . Se presentan 18 secuencias, con tres ítems en cada una de ellas, en las que se deben memorizar desde tres hasta ocho cuadrados.
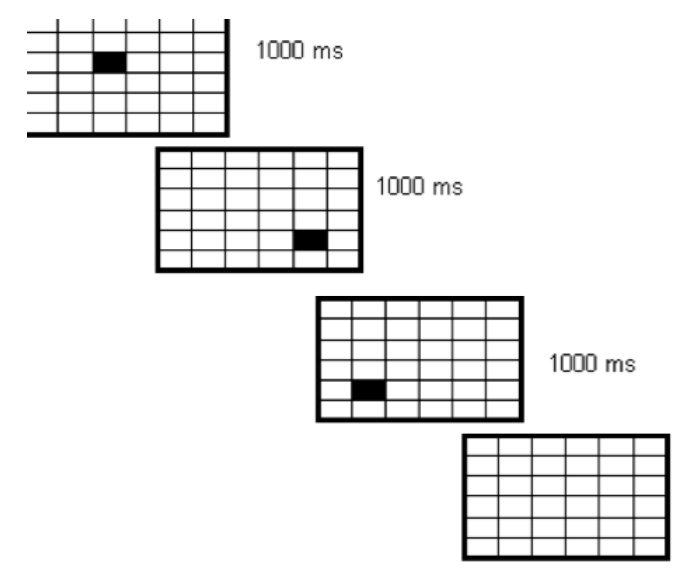

Figura 2. Ejemplo de un ítem de la tarea Espacial. Fuente: Ferreira (2009).

Amplitud de memoria - Dígitos en color: En esta tarea, se presenta individualmente y de forma aleatoria una secuencia de números (0-9) (1000 $\mathrm{ms})$. Cada número muestra un color diferente (azul, negro y rojo) que debe ser memorizado. A continuación, el sujeto debe colocar en tres cajas diferentes (azul, negro y rojo) el número con el color correspondiente. El sujeto tiene que retener entre dos y seis números, lo que totaliza 20 secuencias con cuatro series del mismo tamaño en cada una de ellas.

Memoria de trabajo - Letras y Números: Tarea similar a la del test de Secuencia de Números y Palabras de la Wechsler Memory Scale (2004), en la que se presenta un conjunto de números (0-9) y letras (A-Z) de forma aleatoria $(1000 \mathrm{~ms})$. A continuación, se le pide al sujeto que organice los números en orden ascendente y las letras en orden alfabético, escribiéndolos en el lugar indicado. Existen dos ejercicios de práctica (dos números / dos letras y tres números / tres letras). La tarea se inicia con una secuencia de dos números y dos letras hasta cuatro números y cuatro letras (18 secuencias).

Memoria de trabajo - Viso-espacial: Basada en la Matriz de Puntos (Kane et al., 2004; Law, Morrin $\&$ Pellegrino, 1995), cada secuencia consiste en una matriz simétrica $6 \times 6$, en la que a los participantes se les pide que identifiquen en la matriz B, la posición simétrica de un "muñeco de nieve" que observaron en la matriz A (tarea distractora). Previamente a esta tarea, aparece una matriz 5 × 5 


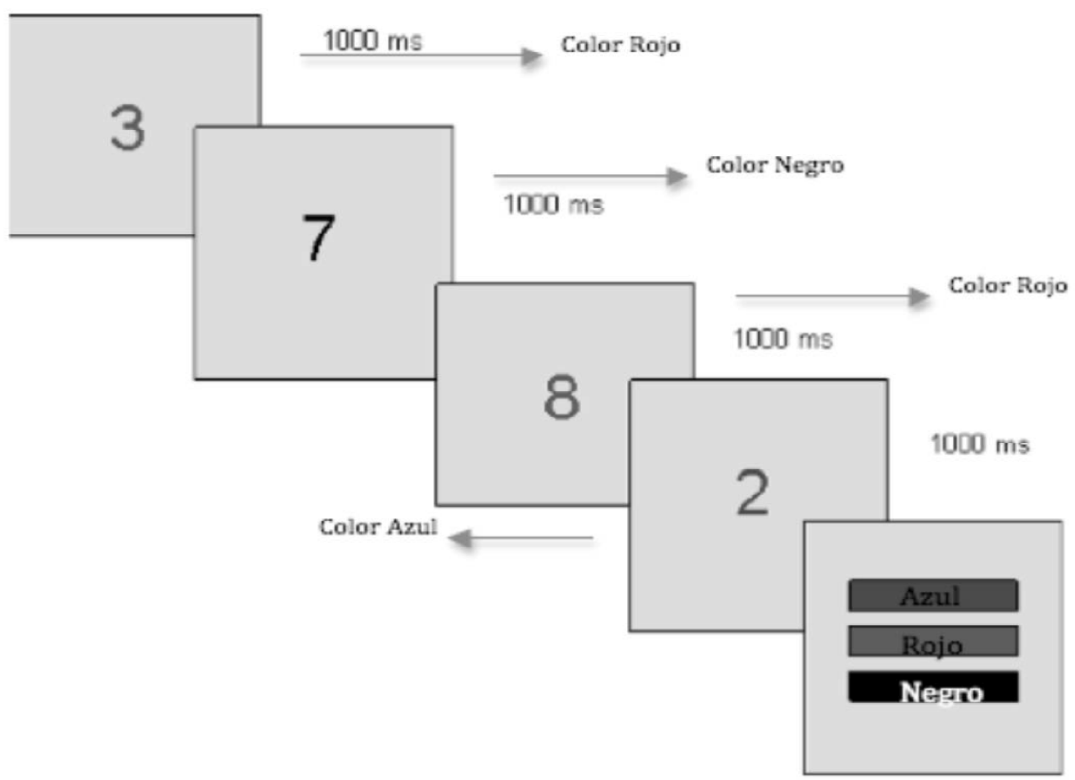

Figura 3. Ejemplo de un ítem de la tarea Dígitos en color. Fuente: Ferreira (2009).

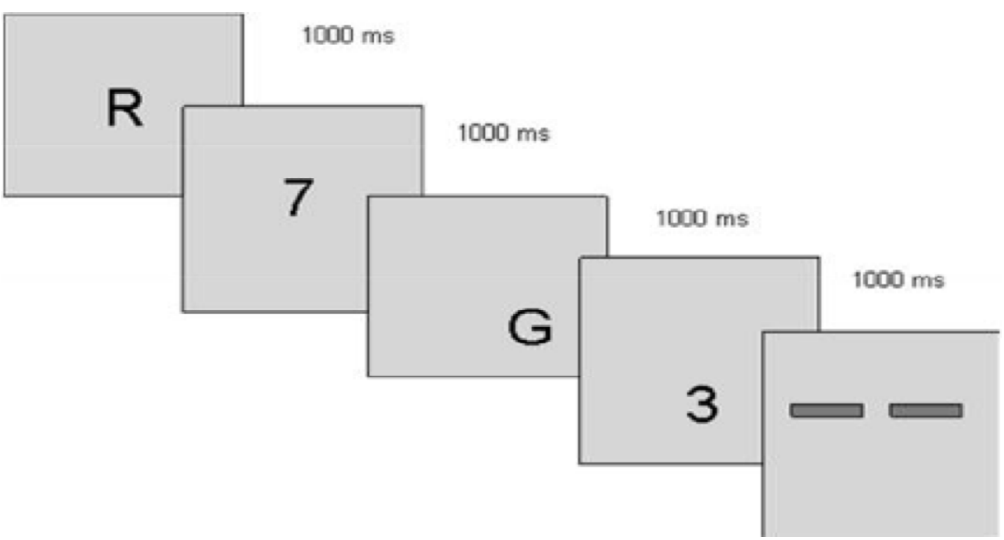

Figura 4. Ejemplo de un ítem de la tarea Letras y Números. Fuente: Ferreira (2009).

durante 1500 ms en la que se presenta un cuadrado negro, cuya ubicación debe ser memorizada. Después de haber memorizado una secuencia de dos a cinco matrices con cuadrados negros, intercaladas con matrices simétricas, se les pide a los sujetos que recuerden el orden directo de presentación de los cuadrados negros. Existen dos ejercicios de práctica con dos matrices simétricas y dos matrices con cuadrados. La tarea se inicia con una secuencia de dos cuadrados negros y aumenta progresivamente de dificultad hasta cinco cuadrados, lo que totaliza 20 secuencias. 


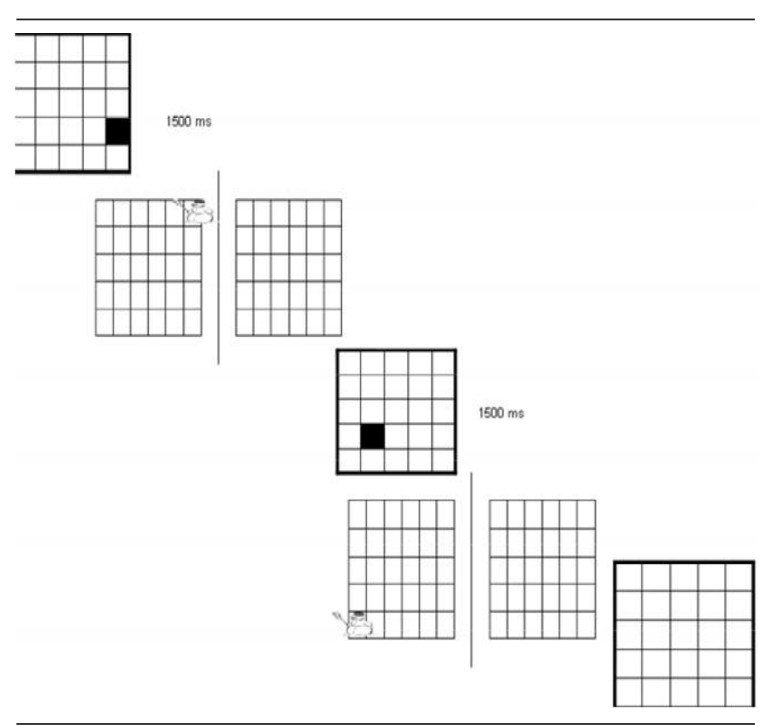

Figura 5. Ejemplo de un ítem de la tarea Viso-espacial. Fuente: Ferreira (2009).

Memoria de trabajo - Flechas y Números: Esta tarea comienza con una secuencia de flechas con diferentes orientaciones (para arriba, para abajo, para los dos lados). Los sujetos deben memorizar la secuencia de flechas que surge durante un breve período de tiempo (1800 ms), a continuación, aparece una secuencia de números. Esta secuencia tiene el mismo tamaño que la secuencia de flechas anteriormente presentada (e.g., a una secuencia de tres flechas le sigue una secuencia de tres números). Los sujetos deben memorizar la secuencia de números y asociar los números a las posiciones de las flechas de acuerdo con la siguiente regla: flecha para arriba - número impar; flecha para abajo -número par; flecha para los dos lados - cero. A partir de entonces, deben identificar la localización de la secuencia donde la regla se incumple. Existen dos ejercicios de práctica (tres flechas / números y cinco flechas / números). A continuación, la tarea comienza con una secuencia de tres flechas / números, hasta nueve flechas / números (21 secuencias).

Test de Factor g: Se utilizó el Test D48, también conocido como test de los Dominós, validado para la población portuguesa (Anstey, 1983). La prueba consta de 44 secuencias de piezas de dominó que

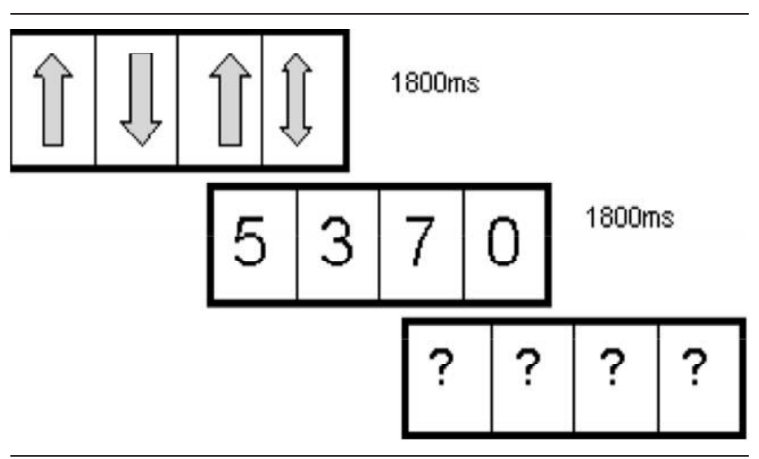

Figura 6. Ejemplo de un ítem de la tarea Flechas y Números. Fuente: Ferreira (2009).

el sujeto debe completar. Los valores de fiabilidad del test oscilan entre 0.78 y 0.99 .

Aptitudes cognitivas: Se utilizaron dos pruebas de la Primary Mental Abilities ([PMA], Thurstone \& Thurstone, 1997) validadas para la población portuguesa: PMA-V formada por ítems de identificación de sinónimos, y PMA-E de identificación de figuras iguales al modelo después de ser rotadas en un espacio bidimensional. La fiabilidad de los resultados en las dos pruebas se sitúa en 0.91 y 0.72 , respectivamente.

\section{Procedimientos}

La aplicación de las pruebas se realizó en dos fases. En una primera fase, los sujetos realizaron, en un orden predeterminado, la batería informatizada de memoria (Dígitos, Espacial, Dígitos en color, Letras y Números, Viso-espacial y Flechas y Números). En una segunda fase, se aplicaron los tres tests de aptitudes (D48, PMA-V y PMA-E), en un formato tradicional de papel y lápiz. El intervalo de tiempo entre las dos sesiones fue de una semana. Los procedimientos de aplicación siguieron las instrucciones presentes en el manual de los tests. Los estudiantes fueron informados de los objetivos del estudio, siendo voluntaria su participación. A aquellos participantes que lo solicitaron, se les proporcionó información sobre su rendimiento en las pruebas. Para el tratamiento de los resultados se utilizaron los paquetes estadísticos SPSS 17.0 y AMOS 6.0. 


\section{Resultados}

Con el objetivo de analizar las relaciones observadas entre las diferentes medidas, se realizó un análisis de correlación de Pearson con los resultados globales obtenidos en las tareas de memoria y de aptitudes / inteligencia. La Tabla 1 permite constatar que existe una correlación significativa entre las diferentes tareas de memoria. En concreto, podemos destacar las correlaciones significativas obtenidas entre tareas con los mismos contenidos (e.g., correlaciones de 0.56 entre Espacial y Viso-espacial y entre Dígitos y Letras y Números). Debemos señalar, también, que el test de inteligencia general (D48) solo muestra correlaciones significativas con los tests de memoria de trabajo Viso-espacial y Flechas y Números. Por su parte, el test de aptitudes verbales (PMA-V) muestra asociaciones significativas con las dos tareas que integran contenidos verbales (Dígitos en color y Letras y Números). A su vez, el test de aptitudes espaciales (PMA-E) está asociado a los dos tests con contenidos espaciales (Espacial y Viso-espacial). Además, aparece fuertemente asociado a la tarea de memoria de trabajo Flechas y Números.

Después de haber encontrado alguna validez de convergencia entre los tests de aptitudes/inteligencia y las pruebas de memoria, se contrastaron dos modelos estructurales que pretendían relacionar diferentes estructuras (amplitud de memoria y memoria de trabajo), diferentes contenidos (verbal y no verbal) y una dimensión de inteligencia general
(Factor g). Así, se propone la asociación de tres tareas a la dimensión latente amplitud de memoria (Dígitos, Espacial y Dígitos en color), y de otras tres con la memoria de trabajo (Letras y Números, Visoespacial, y Flechas y Números). Se plantea también una variable latente para contenidos verbales (Dígitos, Dígitos en color y Letras y Números) y otra para contenidos no verbales (Espacial, Viso-espacial, y Flechas y Números). La variable latente dependiente, denominada Factor $g$, está definida por las variables medidas por el D48 (test de inteligencia general), PMA-V (Verbal) y PMA-E (Espacial).

Debido a que, por una parte, los instrumentos utilizados emplean escalas diferentes y a que, por otra, se observa cierta normalidad en las medidas (asimetría y curtosis), verificada mediante el test de normalidad del AMOS 6.0 (Arbuckle, 2005), se optó por el método de estimación de los Cuadrados Mínimos Ponderados (GLS). Los modelos post hoc justificaron una maximización del ajuste de los modelos con la covariación de los residuos en los tests del PMA (PMA-V y PMA-E) y en las tareas de memoria de trabajo Letras y Números, y Flechas y Números (Figura 7) y en las tareas no verbales Espacial y Viso-espacial (Figura 8).

En una primera fase, se pretendió estudiar la relación entre una variable intermedia asociada al constructo memoria (Gsm) y la inteligencia (Factor g). Este modelo (Figura 7) surge integrado en el modelo $\mathrm{CHC}$, que considera a un nivel superior la variable inteligencia general, y a un nivel interme-

TABLA 1

Medias, desviación típica y correlaciones entre tareas de memoria y test de inteligencia (D48) y aptitudes (PMA-V/E)

\begin{tabular}{lcccccccccc}
\hline & $M$ & $D E$ & 1 & 2 & 3 & 4 & 5 & 6 & 7 & 8 \\
1. Dígitos & 34.24 & 8.93 & & & & & & & & \\
2. Espacial & 31.11 & 8.42 & $0.39^{*}$ & & & & & & & \\
3. Dígitos en color & 31.67 & 5.98 & $0.47^{*}$ & $0.39^{*}$ & & & & & & \\
4. Letras y Números & 17.68 & 5.23 & $0.56^{*}$ & $0.36^{*}$ & $0.49^{*}$ & & & & & \\
5. Viso-espacial & 22.64 & 8.59 & $0.39^{*}$ & $0.56^{*}$ & $0.41^{*}$ & $0.49^{*}$ & & & & \\
6. Flechas y Números & 11.57 & 2.54 & $0.34^{*}$ & $0.38^{*}$ & $0.35^{*}$ & $0.30^{*}$ & $0.44^{*}$ & & & \\
7. D48 & 28.75 & 6.26 & 0.01 & 0.19 & 0.03 & 0.12 & $0.34^{* *}$ & $0.25^{*}$ & & \\
8. PMA-V & 24.41 & 7.44 & 0.09 & -0.05 & $0.24^{*}$ & $0.23^{*}$ & 0.14 & 0.13 & 0.17 & \\
9. PMA-E & 25.75 & 13.15 & 0.07 & $0.28^{*}$ & 0.04 & 0.03 & $0.33^{* *}$ & $0.34^{* *}$ & $0.37^{* *}$ & 0.04 \\
\hline
\end{tabular}

$* p<0.05 ; * * p<0.01$

Fuente: elaboración propia. 


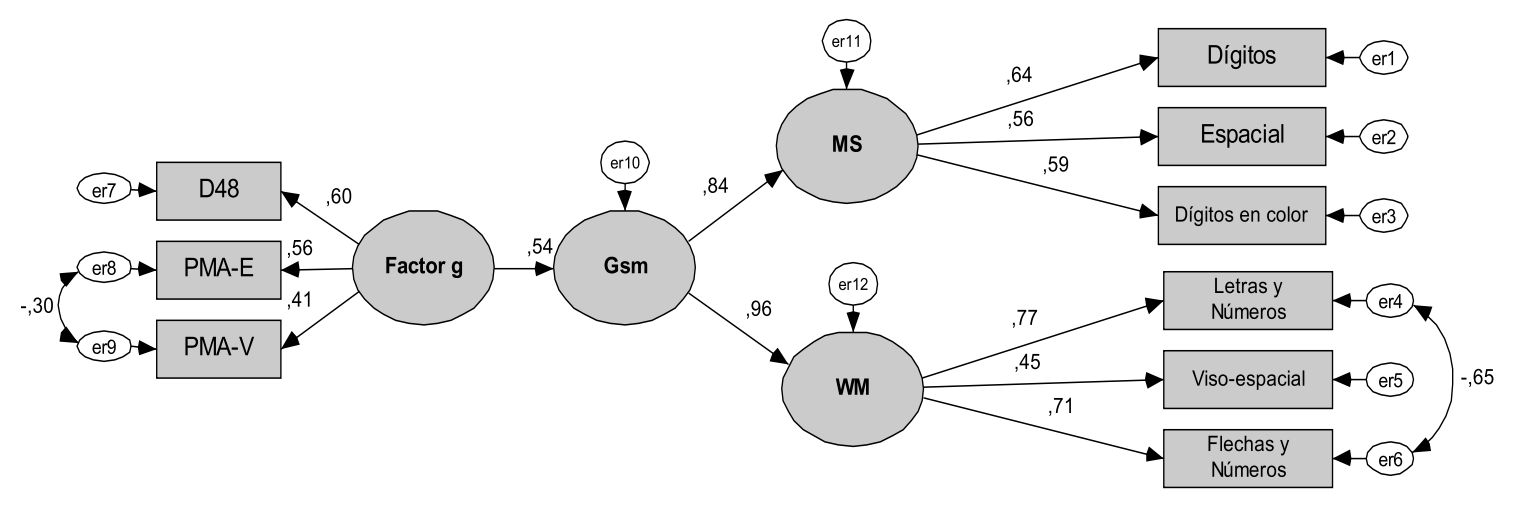

Figura 7. Estructura jerárquica de la memoria y su relación con el Factor g de acuerdo con el modelo CHC.

Fuente: elaboración propia.

dio el constructo memoria (Gsm), asociado, a su vez, a aptitudes de nivel inferior que remiten hacia las tareas de Amplitud de Memoria (Dígitos, Espacial y Dígitos en color), así como, hacia las tareas de Memoria de Trabajo (Letras y Números, Viso-espacial, y Flechas y Números).

Los resultados observados muestran un ajuste de los datos con una aproximación del chi cuadrado a los grados de libertad, e índices de ajuste encuadrados en los valores defendidos en la literatura $\left(x_{(23)}^{2}=22.913, p=0.466 ; \mathrm{CFI}=1.00\right.$; $\mathrm{TLI}=1.007 ; \mathrm{RMSEA}=0.000 ; \mathrm{LO}=0.000$; $\mathrm{HI}=0.097)$. El modelo muestra datos de regresión estandarizados con una regresión positiva entre el constructo memoria (Gsm) y los respectivos subcomponentes MS $(0.84, p<0.001)$ y WM $(0.96$, $p<0.001)$. También se debe destacar el valor de regresión entre el constructo memoria y el componente general de la inteligencia $(0.54, p<0.001)$.

A continuación, existiendo en nuestro estudio tareas con contenidos específicos, se procuró modelar dos variables latentes para contenidos verbales y no verbales, y analizar su relación con una dimensión intermedia de Gsm asociada al factor general de inteligencia de acuerdo con el modelo CHC. El modelo estructural descrito en la Figura 8 se mostró ajustado $\left(\mathrm{X}_{(23)}^{2}=22.3, p=0.506\right.$; $\mathrm{CFI}=1.000$; $\mathrm{TLI}=1.024$; $\mathrm{RMSEA}=0.000$; $\mathrm{LO}=0.000 ; \mathrm{HI}=0.094$ ), permitiendo afirmar que las tareas de memoria con contenidos no verbales aportan una mayor contribución a la dimensión Gsm $(0.98, p<0.001)$ que las tareas de memoria con contenidos verbales $(0.60, p<0.001)$. El valor de regresión entre el constructo memoria y el componente general de la inteligencia $(0.73, p<0.001)$ es más elevado que el observado en el modelo 1.

\section{Discusión y conclusión}

En este estudio se intentó responder a dos preguntas. En primer lugar, se pretendió determinar si la diferenciación del constructo Gsm, dentro del segundo estrato del modelo CHC, se manifiesta mejor en función de los procesos (amplitud de memoria y memoria de trabajo) o en función de los contenidos (verbales y no verbales). En segundo lugar, se procuró analizar la relación entre la aptitud intermedia de Gsm con una aptitud general de inteligencia.

Tal como se hipotetiza en el modelo CHC de las aptitudes humanas, las tareas de memoria surgen asociadas a una aptitud de nivel intermedio - Gsm, que a su vez refleja un factor de dominio general. De acuerdo con estudios previos (Engle et al., 1999), es posible verificar un ajuste en los modelos 1 y 2 que confirma la relación jerárquica del constructo Memoria, semejante al propuesto en el modelo $\mathrm{CHC}$.

Además, se procuró comprender si Gsm, en cuanto aptitud intermedia del modelo, muestra un mejor ajuste cuando la diferenciamos en función de los procesos (MS, amplitud de memoria y WM, memoria de trabajo) o en función de los contenidos (verbales y no verbales). Ambos modelos se ajustan, aunque el modelo 2 presenta un mejor ajuste, si bien mínimo, de los datos. 


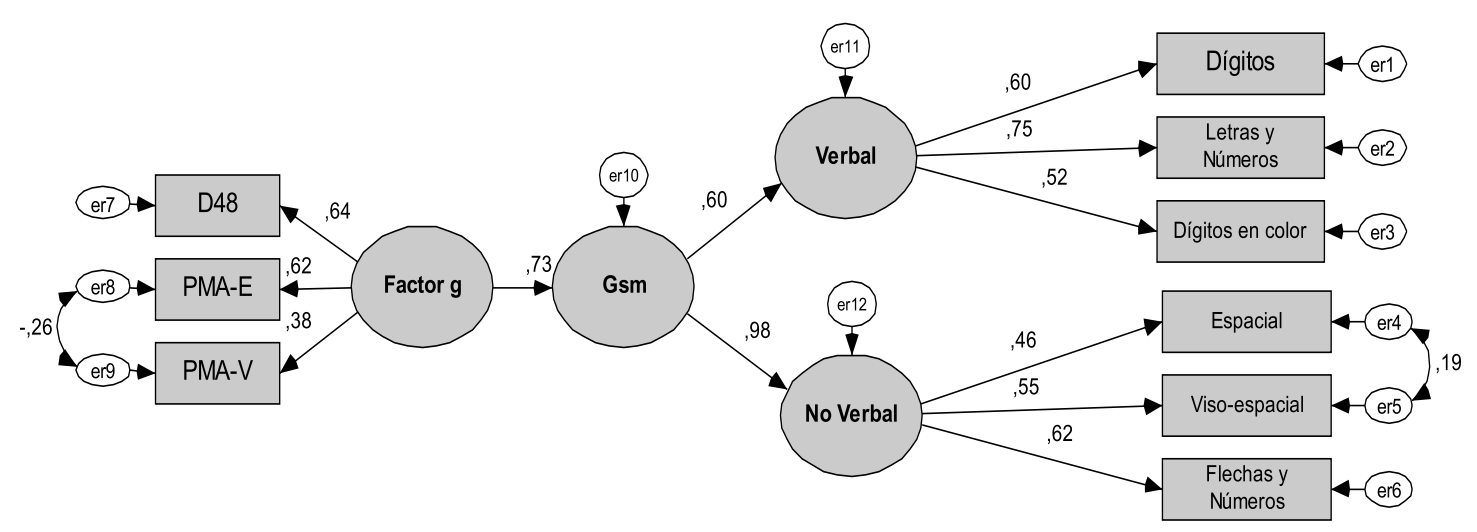

Figura 8. Estructura jerárquica de los contenidos de la memoria y su relación con el Factor g de acuerdo con el modelo CHC. Fuente: elaboración propia.

Como se puede observar en el modelo 1, la relación entre memoria de trabajo y Gsm aumenta hasta 0.96, mientras que la relación entre la amplitud de memoria y Gsm disminuye hasta 0.84, ambos valores muy altos y significativos, que ponen de manifiesto la independencia de los constructos en su relación con el factor intermedio Memoria. Estos resultados están de acuerdo con otros estudios que sugieren la independencia de los factores amplitud de memoria y memoria de trabajo (Bayliss et al., 2003; Kane et al., 2004). Cuando se analizó el mayor impacto de la memoria de trabajo en Gsm, se podría señalar que las funciones ejecutivas inherentes a las tareas de memoria de trabajo justifican un aumento de las correlaciones.

En el modelo 2, la relación entre tareas con contenido no verbal y Gsm es muy elevada (0.98), disminuyendo esta relación de manera significativa con tareas con contenido verbal (0.60). Esta separación de Gsm en tareas con contenidos verbales y no verbales también ha sido confirmada en otros estudios (Baddeley \& Hitch, 1974; Engle, Kane et al., 1999; Ferreira, 2009; Shah \& Miyake, 1996). La mayor asociación de los contenidos no verbales con Gsm podría deberse a una mayor contribución en el factor $g$ de los contenidos viso-espaciales de la memoria que de los contenidos verbales. Por ejemplo, en el estudio realizado por Kane et al. (2004), se concluyó que las correlaciones entre contenidos no verbales eran de 0.54 , mientras que las correlaciones de los contenidos verbales eran solo de 0.16. Estos datos pueden explicar el hecho de que los tests de inteligencia general incluyan tareas con figuras no verbales.

Los datos muestran también que la relación entre inteligencia general y el constructo Gsm aparece con un valor superior de coeficiente de regresión en el modelo 2 (0.73) que en el modelo 1 (0.54). Estos resultados sugieren que la influencia de $g$ es mayor cuando Gsm se define con base en contenidos que cuando se lleva a cabo recurriendo a procesos. Sin embargo, estos dos valores son manifiestamente superiores al coeficiente de regresión de 0.29 obtenido en estudios previos (Engle et al., 1999). Los valores del coeficiente de regresión logrados en el presente estudio son también ligeramente superiores al valor de 0.48 mostrado en el metaanálisis de Ackerman et al. (2005). La correlación existente entre factor $g$ y Gsm va en la línea de los diferentes estudios en neurociencias. En este sentido, la correlación que se distancia de la unidad garantiza la autonomía funcional del córtex cerebral a nivel del funcionamiento del factor $g$ (Gläscher et al., 2010). Por otro lado, las correlaciones significativas sugieren la proximidad de los constructos pudiendo compartir las mismas áreas del cerebro (Colom et al., 2007; Garlick \& Sejnowski, 2006; Prabhakaran et al., 1997). Así, 
se puede señalar que los resultados obtenidos no son tan elevados como los de Kyllonen y Christal (1990), pero se aproximan a los resultados de 0.60 (Conway, Cowan, Bunting, Therriault \& Minkoff, 2002), 0.59 (Engle et al., 1999), 0.64 y 0.52 (Kane et al., 2004) y 0.67 (Schweizer, 2007) de otros estudios. Aunque alguna literatura (e.g., Kane et al., 2004) señala la posibilidad de establecer 0.60 \pm 0.05 como el número mágico de la correlación de la memoria con $g$, se plantea que este intervalo podría ser significativamente mayor (e.g., $0.60 \pm$ 0.10 ), debido a que las características de las tareas y el número de variables incluidas en el estudio, junto con el error de las variables latentes, podrían alterar los resultados de las correlaciones obtenidas.

La inclusión de tareas nuevas y de una muestra con un número mayor de sujetos deberían ser aspectos a salvaguardar en futuras investigaciones. Una limitación importante de este estudio es el hecho de que la dimensión general de inteligencia es el resultado de tres tests bastante diferenciados, que presentan correlaciones bajas entre sí (0.17 y 0.37). En este sentido, sería importante introducir tareas adicionales de inteligencia que evaluasen toda la complejidad del constructo. Si se parte de la evidencia que muestra la fuerte contribución de la inteligencia fluida y cristalizada en el factor $g$ (Carroll, 1993; Cattell, 1971; Hunt, 1999), se podría considerar la inclusión en un nuevo estudio de otras tareas que abordasen el conocimiento cultural, el razonamiento viso-espacial y la aptitud para razonar rápidamente. En la línea de un estudio realizado por Martínez y Colom (2009), sería interesante incluir otras tareas fuertemente representativas de la inteligencia general, como $\mathrm{G} v$, asociadas al componente de aptitud visual. No obstante, se considera que este estudio introduce algunas cuestiones que destacan la importancia de las funciones ejecutivas (memoria de trabajo) y los contenidos no verbales de la memoria en su relación con $g$. De alguna forma, se destaca el hecho de que el factor Gsm del modelo CHC puede ser diferenciado en función de los contenidos y procesos de la memoria, siendo los contenidos responsables de una mayor varianza explicativa de la inteligencia.

\section{Referencias}

Ackerman, P. L., Beier, M. E. \& Boyle, M. O. (2005). Working memory and intelligence: The same or different constructs? Psychological Bulletin, 131, 30-60.

Almeida, L. S. (1994). Inteligência: Definição e medida. Aveiro: Centro de Investigação Educacional, Universidade de Aveiro.

Almeida, L. S., Guisande, M. A. \& Ferreira, A. I. (2009). Inteligência: Perspectivas teóricas. Coimbra: Almedina.

Anstey, E. (1983). Dominoes 48. Lisboa: CEGOC, TEA.

Arbuckle, J. L. (2005). AMOS 6.0 User's Guide. Chicago, IL: SPSS Inc.

Baddeley, A. D. \& Hitch, G. J. (1974). Working memory. En G. H. Bower (Ed.), The psychology of learning and motivation (Vol. 8, pp. 47-89). New York: Academic Press.

Bayliss, D. M., Jarrold, C., Gunn, D. M. \& Baddeley, A. D. (2003). The complexities of complex span: Explaining individual differences in working memory in children and adults. Journal of Experimental Psychology: General, 132(1), 71-92.

Carroll, J. B. (1993). Human cognitive abilities. Cambridge, MA: Cambridge University.

Cattell, R. B. (1971). Abilities: Their structure, growth, and action. Boston: Houghton Mifflin.

Colom, R., Abad, F. J., Rebollo, I. \& Shih, P. C. (2005). Memory span and general intelligence: A latent variable approach. Intelligence, 33, 623-642.

Colom, R. \& Flores-Mendoza, C. (2006). Armazenamento de curto prazo e velocidade de processamento explicam a relação entre memória de trabalho e o factor $g$ de inteligência. Psicologia: Teoria e Pesquisa, 22, 113-122.

Colom, R., Flores-Mendoza, C. \& Rebollo, I. (2003). Working memory and intelligence. Personality and Individual Differences, 34, 33-39.

Colom, R., Jung, R. E. \& Haier, R. J. (2007). General intelligence and memory span: Evidence for a common neuroanatomic framework. Cognitive Neuropsychology, 24, 867-878.

Colom, R., Rebollo, I., Palácios, A., Juan-Espinosa, M. \& Kyllonen, P. (2004). Working memory is (almost) perfectly predicted by g. Intelligence, 32, 277-296. 
Colom, R., Rubio, V. J., Shih, P. C. \& Santacreu, J. (2006). Fluid intelligence, working memory and executive functioning. Psicothema, 18, 819-821.

Conway, A. R., Cowan, N., Bunting, M., Therriault, D. \& Minkoff, S. (2002). A latent variable analysis of working memory capacity, short-term memory capacity, processing speed, and general fluid intelligence. Intelligence, 30, 163-183.

Engle, R. W., Kane, M. J. \& Tüholski, S. W. (1999). Individual differences in working memory capacity and what they tell us about controlled attention, general fluid intelligence, and functions of the prefrontal cortex. En A. Miyake \& P. Shah (Eds.), Models of working memory: Mechanisms of active maintenance and executive control (pp. 102-134). Cambridge: Cambridge University Press.

Engle, R. W., Tüholski, S. W., Laughlin, J. \& Conway, A. R. A. (1999). Working memory, short-term memory, and general fluid intelligence: A latentvariable approach. Journal of Experimental Psychology: General, 128, 309-331.

Ferreira, A. I. (2009). Perspectiva multifactorial da memória: Construção e validação de uma bateria de provas para a população universitária portuguesa. Tesis de doctorado no publicada, Universidade do Minho, Braga, Portugal.

Ferreira, A. I., Almeida, L. S. \& Prieto, G. (2011). The role of processes and contents in human memory: An item response theory approach. Journal of Cognitive Psychology, 23, 873-885.

Garlick, D. \& Sejnowski, T. J. (2006). There is more to fluid intelligence than working memory capacity and executive function. Behavioral and Brain Sciences, 29, 134-135.

Gläscher, J., Rudrauf, D., Colom, R., Paul, L. K., Tranel, D., Damasio, H., et al. (2010). Distributed neural system for general intelligence revealed by lesion mapping. Proceedings of the National Academy of Sciences, 107, 4705-4709.

Guilford, J. P. (1967). The nature of human intelligence. New York: McGraw-Hill.

Horn, J. L. \& Cattell, R. B. (1966). Refinement and test of the theory of fluid and crystallized ability intelligence. Journal of Educational Psychology, 57, 253-270.
Horn, J. L. \& Noll, J. C. (1997). Human cognitive capabilities: Gf-Gc theory. En D. P. Flanagan, J. L. Genshaft \& P. L. Harrison (Orgs.), Contemporary intellectual assessment: Theories, tests, and issues (pp. 53-91). New York: Guilford Press.

Hunt, E. (1999). Intelligence and human resources: Past, present and future. En P. L. Ackerman, P. C. Kyllonen \& R. D. Roberts (Eds.), Learning and individual differences (pp. 3-30). Washington, DC: APA.

Ichikawa, S. (1983). Verbal memory span, visual memory span, and their correlations with cognitive tasks. Japanese Psychological Research, 25, 173-180.

Jensen, A. R. (1973). Level I and Level II abilities in three ethnic groups. American Educational Research Journal, 10, 263-276.

Jensen, A. R. (1998). The $g$ factor: The science of mental ability (Human evolution, behavior, and intelligence). Westport, CT: Praeger.

Kane, M. J., Hambrick, D. Z., Wilhelm, O., Payne, T., Tüholski, S. \& Engle, R. W. (2004). The generality of working memory capacity: A latent variable approach to verbal and visuo-spatial memory span and reasoning. Journal of Experimental Psychology: General, 133, 189-217.

Kyllonen, P. C. \& Christal, R. E. (1990). Reasoning ability is (little more than) working-memory capacity?! Intelligence, 14, 389-433.

Law, D. J., Morrin, K. A. \& Pellegrino, J. W. (1995). Training effects and working memory contributions to skill acquisition in a complex coordination task. Learning and Individual Differences, 7, 207-234.

Martínez, K. \& Colom, R. (2009). Working memory capacity and processing efficiency predict fluid but not crystallized and spatial intelligence: Evidence supporting the neural noise hypothesis. Personality and Individual Differences, 46, 281-286.

Milner, B. (1971). Interhemispheric differences in the localization of psychological processes in man. British Medical Bulletin, 27, 272-277.

Oberauer, K., Schulze, R., Wilhelm, O. \& Süß, H. M. (2005). Working memory and intelligence--their correlation and their relation: Comment on Ackerman, Beier, and Boyle (2005). Psychological Bulletin, 131(1), 61-65.doi:10.1037/0033-2909.131.1.61 
Prabhakaran, V., Smith, J. A., Desmond, J. E., Glover, G. H. \& Gabrieli, J. D. (1997). Neural substrates of fluid reasoning: An fMRI study of neocortical activation during performance of the Raven's Progressive Matrices Test. Cognitive Psychology, 33, 43-63.

Schweizer, K. (2007). Investigating the relationship of working memory tasks and fluid intelligence tests by means of the fixed-links model in considering the impurity problem. Intelligence, 35, 591-604.

Shah, P. \& Miyake, A. (1996). The separability of working memory resources for spatial thinking and language processing: An individual differences approach. Journal of Experimental Psychology: General, 125, 4-27.

Spearman, Ch. (1904). "General Intelligence" objectively determined and measured. American Journal of Psychology, 15, 201-293.
Spearman, Ch. (1927). The abilities of man: Their nature and measurement. New York: MacMillan.

Sternberg, R. J. (1977). Component processes in analogical reasoning. Psychological Review, 84, 353-378.

Thurstone, L. L. (1938). Primary mental abilities. Chicago: University of Chicago Press.

Thurstone, L. L. \& Thurstone, T. G. (1997). Primary Mental Abilities. Lisboa: CEGOC, TEA.

Unsworth, N. \& Engle, R. N. (2005). Working memory capacity and fluid abilities: Examining the correlations between operation span and Raven. Intelligence, 33, 67-81.

Vernon, P. E. (1961). The measurement of abilities. Oxford: Philosophical Library.

Wechsler, D. (2004). Wechsler Intelligence Scales for Children IV. London: Psychological Corporation. 
